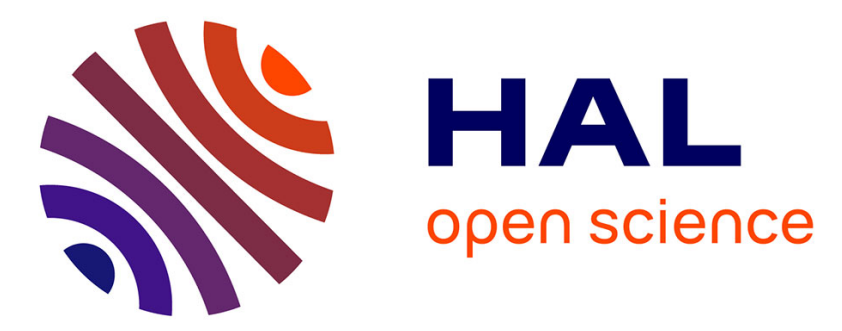

\title{
Tuning the magnetic properties of beryllium chains
}

\author{
Stefano Battaglia, Noelia Faginas Lago, Thierry Leininger, Stefano Evangelisti
}

\section{To cite this version:}

Stefano Battaglia, Noelia Faginas Lago, Thierry Leininger, Stefano Evangelisti. Tuning the magnetic properties of beryllium chains. Physical Chemistry Chemical Physics, 2019, 21, pp.6080-6086. 10.1039/C8CP07159D . hal-02128292

\section{HAL Id: hal-02128292 \\ https://hal.science/hal-02128292}

Submitted on 22 Oct 2019

HAL is a multi-disciplinary open access archive for the deposit and dissemination of scientific research documents, whether they are published or not. The documents may come from teaching and research institutions in France or abroad, or from public or private research centers.
L'archive ouverte pluridisciplinaire HAL, est destinée au dépôt et à la diffusion de documents scientifiques de niveau recherche, publiés ou non, émanant des établissements d'enseignement et de recherche français ou étrangers, des laboratoires publics ou privés. 


\section{Journal Name}

\section{ARTICLE TYPE}

Cite this: DOI: $10.1039 / x x x x x x x x x x$

Accepted Date

DOI: 10.1039/xxxxxxxxxx

www.rsc.org/journalname

\section{Tuning the magnetic properties of beryllium chains ${ }^{\dagger}$}

\author{
Stefano Battaglia, ${ }^{a, b}$ Noelia Faginas-Lago*,$b$ Thierry Leininger ${ }^{a}$ and Stefano \\ Evangelisti ${ }^{* a}$
}

In this work we explore the effect of confining beryllium chains inside carbon nanotubes. Linear $\mathrm{Be}_{n}$ systems are characterized by two states originating from the presence of edge orbitals localized at the chain extremities. The two spins occupying these orbitals are, in gas phase, antiferromagnetically coupled, with the magnetic coupling $J$ decaying exponentially as a function of the increasing length of the chain. When inserted into narrow carbon nanotubes, the linear geometry is found to be more stable than the more compact cluster conformation favored for the isolated case: the lack of space inside the cavity prevents the chain to fold. Most importantly, the presence of the surrounding nanotube does not only preserve the linear structure of $\mathrm{Be}_{n}$, but it affects its magnetic properties too. In particular it was found that the magnetic coupling between the ground and the first excited state can be modulated according to the nanotube diameter as well as chain length, and our calculations suggest a possible direct relationship between these parameters and $J$. This behavior can be exploited to engineer a composite $\mathrm{Be}_{n} @ \mathrm{CNT}$ system with a magnetic coupling tuned by construction, with interesting potential applications.

\section{Introduction}

Since their discovery in 1991 by Iijima ${ }^{1}$, carbon nanotubes (CNTs) have been subject to an incredible amount of investigations due to their potential applications in a wide array of domains such as electronics, biology, information technology and medical sciences, to name a few 2 . Because of their hollow structure, a virtually infinite number of composite materials can be created by inserting different types of molecules into their cavity. Indeed, already in 1993, Ajayan and the same Iijima showed experimentally the possibility to fill CNTs by capillary suction, proving the feasibility of early theoretical predictions ${ }^{3}$. Since then, a lot of work has been carried out in this direction, both at experimental and theoretical levels, such that CNTs have been used to host a large variety of systems such as fullerenes and fullerenebased molecules $\frac{45}{5}$, metals $\sqrt{6-12}$, energetic compounds $\frac{13}{16}$, and many more.

The encapsulation of molecular systems inside CNTs can alter the chemical properties of the confined species or induce new phases of materials, leading to, sometimes, surprising results. A notable

\footnotetext{
${ }^{a}$ Laboratoire de Chimie et Physique Quantiques, Université de Toulouse et CNRS, 118 Route de Narbonne, F-31062 Toulouse, France. Tel: +33 (0)561 5576 94; E-mail: stefano@irsamc.ups-tlse.fr

${ }^{b}$ Dipartimento di Chimica, Biologia e Biotecnologie, Università degli Studi di Perugia, Via Elce di Sotto 8, I-06123 Perugia, Italy. Tel: +39 07558555 27; E-mail: noelia.faginaslago@unipg.it

$\dagger$ Electronic Supplementary Information (ESI) available: Complete set of parameters used in the calculations. See DOI: 10.1039/b000000x/
}

example is that of the confinement of water, for which new phases were predicted almost 20 years ago by computational studies 17 , subsequently confirmed experimentally 18 and still under active investigation these days 19 .

Considering the quasi one-dimensional structure of nanotubes, linear molecules, nanowires and alike are ideal candidates for confinement. For instance, experimental evidence of linear polyyne chains encaged in single-wall CNTs was reported some time ago 20 , while very recently the polymerization of white phosphorus was observed ${ }^{21}$ by transmission electron microscopy. These studies highlight two remarkable properties of nanotubes: on one side their action as "nanoreactors" favoring the formation of novel structures inside the cavity and on the other side their potential as storage devices preventing the enclosed substances to react, decompose or fold.

This latter property is of particular interest for linear chains of beryllium atoms, whose theoretically predicted structure could potentially be preserved inside the cavity of CNTs. It is in fact known that the global minimum of the potential energy surface (PES) of $\mathrm{Be}_{n}$ molecules corresponds to compact cluster conformations, at least for the first few systems with 6 or less atoms 22 . The interest in preserving the linear geometry lies in the properties of beryllium chains. By sharing two electrons each, beryllium atoms form single covalent bonds with each other and bind in a linear arrangement which leaves two unpaired electrons at the extremities. It was predicted by ab initio calculations that two half-filled orbitals localize at the ends of the chain, giving rise 
to an antiferromagnetically coupled ground state and a low-lying triplet state very close in energy 23 . Moreover, in a subsequent investigation it was found that the strength of the magnetic coupling changes when the chain is deposited on a graphene nanoisland, showing a strong dependence on the distance from the surface 27 . Most importantly, the ground state of the system switches from antiferromagnetic to ferromagnetic for a certain range of distances. It thus appears that the magnetic properties can be tuned by non-covalent interactions with carbon-based nanostructures and there is no reason to believe that a different behavior is observed for the interaction with CNTs. Confinement inside carbon nanotubes has therefore two purposes: preserve the linear conformation of $\mathrm{Be}_{n}$ chains and use their diameter as tuning parameter for the coupling strength, potentially creating a range of hybrid composites with applications, for instance, in the field of molecular magnets.

In this contribution we explore the effects of confining beryllium chains of different lengths inside several carbon nanotubes using both multireference wave function methods as well as density functional theory. On one hand we calculate the energetics of beryllium chains and compare them to their cluster counterparts, and on the other hand we investigate the magnetic coupling of the chains as a function of both the length of the chain and the diameter of the nanotube.

The article is organized as follows. In the next section we present all the details regarding the methodology used in this work. This is followed by two sections presenting the results obtained in this work, in particular we first provide a comparison of linear and cluster conformations outside and inside CNTs and subsequently report the main findings regarding the magnetic properties of the composite systems. In the Conclusions section we summarize and close the discussion.

\section{Computational Details}

In this work we considered two beryllium chains, $\mathrm{Be}_{n}$, with $n=4,5$, which were encapsulated inside finite-size carbon nanotubes of different diameters.

The ground state of the isolated chains is the ${ }^{1} \Sigma_{g}$ singlet state irrespective of their length and it is always of open-shell character 23.26. To correctly describe this type of wave function without breaking the spin symmetry and obtain accurate values of the magnetic coupling, a multireference approach is required. Therefore, the calculations to compute the magnetic coupling were carried out using the complete active space selfconsistent field (CASSCF) method 28 , using an active space composed by two electrons in two orbitals, the latter being localized on both terminal atoms of the beryllium chain as exemplified in Figure 1 for $\mathrm{Be}_{5}$. Dynamical electron correlation was included through second-order $n$-electron valence perturbation theory (NEVPT2) 29 31, using the CASSCF wave functions as zerothorder states. For all calculations, the correlation-consistent basis set family by Dunning et al. $\frac{32}{32}$ was used, in particular the cc-pVTZ basis for beryllium and the smaller double- $\zeta$ cc-pVDZ basis for the nanotube.

The geometry of the isolated chains was optimized at NEVPT2/ccpVTZ level of theory for both the singlet ${ }^{1} \Sigma_{g}$ and the triplet ${ }^{3} \Sigma_{u}$

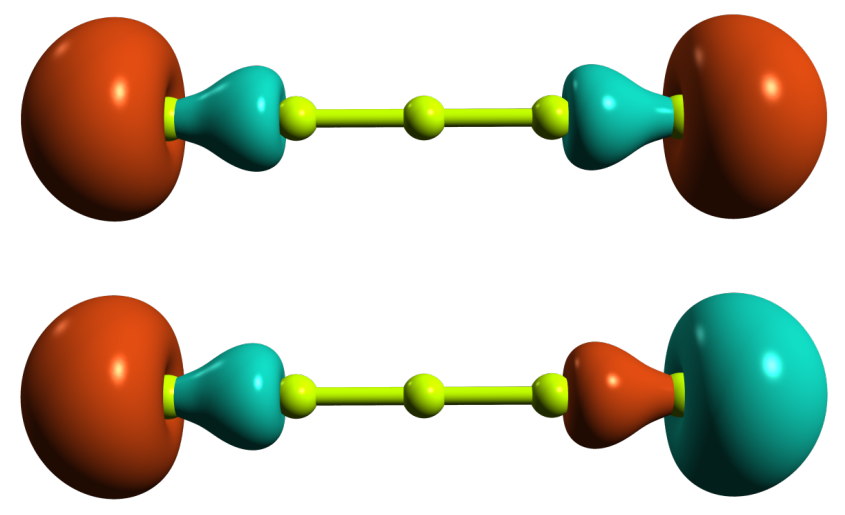

Fig. 1 Edge molecular orbitals included in the active space.

states separately. The bond lengths obtained are in good agreement with previous investigations 23,26 and are reported in Table 1. Given the similar bond lengths for both states, we have

Table 1 Beryllium chains bond lengths in $\AA$

\begin{tabular}{cccc}
\hline system & state & $\begin{array}{c}\text { Internal } \\
\text { bond length }\end{array}$ & $\begin{array}{c}\text { External } \\
\text { bond length }\end{array}$ \\
\hline \multirow{2}{*}{$\mathrm{Be}_{4}$} & ${ }^{1} \Sigma_{g}$ & 2.110 & 2.124 \\
& ${ }^{3} \Sigma_{u}$ & 2.109 & 2.122 \\
\hline \multirow{2}{*}{$\mathrm{Be}_{5}$} & ${ }^{1} \Sigma_{g}$ & 2.113 & 2.126 \\
& ${ }^{3} \Sigma_{u}$ & 2.111 & 2.123 \\
\hline
\end{tabular}

used the geometry of the singlet state for all calculations carried out in this work. The carbon nanotubes geometries were generated using the nanotube builder provided by the Avogadro software $\frac{33}{3}$, setting the carbon-carbon bond distance to $1.421 \AA$ and the carbon-hydrogen one to $1.032 \AA$. We did not optimize the geometry of the CNTs for two reasons: on one side it is well known that they are very stiff systems, and on the other side we do not expect the fine details of the nanotube geometry to have a strong effect on the beryllium chain. In this study we considered CNTs with four different diameters, whose structural parameters are listed in Table 2. Since the two $\mathrm{Be}_{n}$ chains are of different

Table 2 Structural parameters of the CNTs used in this work. The first column reports the chiral indices of the CNTs

\begin{tabular}{ccc}
\hline$(m, l)$ & diameter $[\AA]$ & lengths $[\AA]$ \\
\hline$(5,4)$ & 6.12 & 17.16 \\
$(5,5)$ & 6.78 & 16.01 \\
$(6,5)$ & 7.47 & 17.06 \\
$(6,6)$ & 8.14 & 16.00 \\
\hline
\end{tabular}

lengths, the nanotubes length was modeled accordingly. Note that the values reported in Table 2 are computed from the two extremest carbon atoms and not from the hydrogen atoms added at the ends to saturate the nanotube.

To compare the energy of the linear chains with that of the most stable beryllium clusters and to compute the interaction energy 
between the $\mathrm{Be}_{n}$ systems and the CNTs we used unrestricted (broken-symmetry) Kohn-Sham density functional theory (DFT) in combination with the APFD exchange and correlation functional ${ }^{34}$. In this case the spin symmetry could not be preserved, however the spin contamination observed was insignificant. The basis set used for these calculations is the same as before.

For all wave function calculations we used the ORCA program, version 4.0.1.2 $2^{35}$. Given the size of the systems and the basis set used, for both CASSCF and NEVPT2 calculations we relied on the RIJCOSX approximation 36 using the universal def2/JK auxiliary basis set 37 . The complete overview of the computational parameters used for this part is available in the Supporting Information ${ }^{\dagger}$. All DFT calculations were carried out using the Gaussian 09 software $\frac{38}{}$, setting the "ultrafine" integration grid and the keyword "guess $=$ mix" after a suitable reordering of the orbitals.

Single point energies computed for different electronic states were performed in state-specific mode. Note that calculations based on a state-average formalism produced virtually identical results, at least for the isolated chains.

\section{Stability of Beryllium Chains}

In the first part of this manuscript, before discussing the magnetic properties of the systems under investigation, we present the thermodynamic stability of the linear conformation of both $\mathrm{Be}_{4}$ and $\mathrm{Be}_{5}$ towards the most stable geometry, as well as the interaction energy of the former with the hosting carbon nanotube for a few selected cases. The values listed in Table 3 show the thermodynamic energy differences between the linear structure and the cluster geometry, noting that the latter is known to be the global minimum of the singlet manifold ${ }^{22}$ for both $\mathrm{Be}_{4}$ and $\mathrm{Be}_{5}$. The

Table 3 Summary of electronic energy (with and without zero-point vibrational energy correction), enthalpy and free energy differences between the linear and the cluster geometries. All values are given in $\mathrm{kcal} / \mathrm{mol}$

\begin{tabular}{ccccc}
\hline System & $\Delta E_{e l}$ & $\Delta E_{e l+Z P V}$ & $\Delta H$ & $\Delta G$ \\
\hline $\mathrm{Be}_{4}$ & 56.6 & 54.3 & 55.6 & 52.5 \\
$\mathrm{Be}_{5}$ & 72.8 & 69.8 & 71.5 & 68.0 \\
\hline
\end{tabular}

linear conformation is thermodynamically unstable with repsect to the cluster one, with free energy differences of $52.5 \mathrm{kcal} / \mathrm{mol}$ and $68.0 \mathrm{kcal} / \mathrm{mol}$ for $\mathrm{Be}_{4}$ and $\mathrm{Be}_{5}$, respectively. The increase in energy difference observed between $n=4$ and $n=5$ is likely to continue for higher $n$ values as well: the system is more flexible in the cluster conformation and atoms can rearrange to form several new bonds upon addition of Be atoms, whereas to maintain a linear geometry, each extra atom only forms a new single bond along the chain. Therefore, given this substantial energy difference, the linear conformation appears to be a much more unlike realization than the cluster geometry, at least in the case of an isolated system, with the ultimate consequence of losing the interesting magnetic properties characterizing the chain. An investigation of the potential energy surface would be necessary to identify the isomerization pathways between these structures and the associated kinetics, providing a final answer on the stability of the isolated chain. However, this goes beyond the scope of this study and hence is not carried out further here. Instead, what we propose is to encapsulate the $\mathrm{Be}_{n}$ chain inside narrow carbon nanotubes. The idea is that the limited space available in the cavity prevents the chain to fold and form the cluster structure, effectively blocking all isomerization pathways and preserving the linear conformation. An example of a $\mathrm{Be}_{n} @ \mathrm{CNT}(m, l)$ composite system for $n=m=l=5$ is depicted in Figure 2. In order to eval-
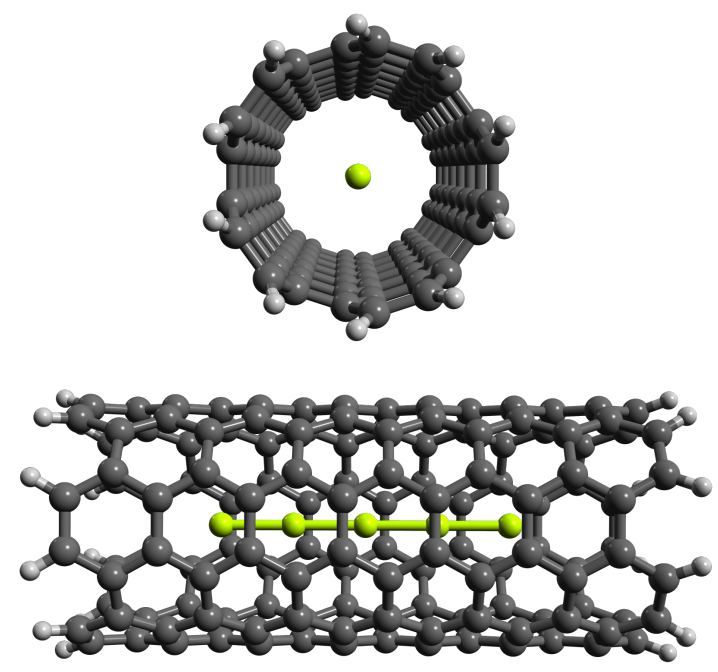

Fig. 2 Front and side view of $\mathrm{Be}_{5} @ \mathrm{CNT}(5,5)$.

uate in first approximation the feasibility of such approach, we calculated the interaction energy of a few selected systems. The values are obtained according to the simple formula

$$
E_{\text {int }}=E_{\text {complex }}-E_{B e_{n}}-E_{C N T}
$$

where the energy of the fragments, i.e. $E_{B e_{n}}$ and $E_{C N T}$, was obtained in the basis of the complex system following the counterpoise correction scheme 39 . To estimate the impact of such approach and test the validity of the model used, we computed the energies of the fragments in their own basis, as well as the interaction energy with a larger cc-pVTZ basis set on the CNT. These calculations were carried out for the systems $\mathrm{Be}_{4} @ \mathrm{CNT}(5,5)$ and $\mathrm{Be}_{4} @ \mathrm{CNT}(6,6)$, with the beryllium fragment being either in the linear or cluster conformation. Note that in the case of the chain, we completely neglected relaxation effects as stated in the preceding section, while for the cluster conformation, $\mathrm{Be}_{n}$ was allowed to relax, but not the nanotube. This choice was taken considering in particular the system $\mathrm{Be}_{4} @ \mathrm{CNT}(5,5)$, where the limited space of the cavity could in principle strongly affect the geometry of the cluster. For all unrelaxed interaction energies, the $\mathrm{Be}_{n}$ fragment was placed in the center of the CNT. The values obtained are reported in Table 4. Let us first consider the results obtained in the narrower nanotube, i.e. the $\operatorname{CNT}(5,5)$. The interaction energy is negative, indicating a favorable adsorption of $\mathrm{Be}_{4}$ inside the CNT and in particular, we see that the interaction of the chain is much stronger than that of the cluster by as much as $\approx 30 \mathrm{kcal} / \mathrm{mol}$, suggesting strong effects of the spatial constraints provided by the nanotube in the case of the cluster. This picture is corroborated by the energy difference between the 
Table 4 Interaction energies (with counterpoise correction) between $\mathrm{Be}_{4}$ and two different CNTs for both the linear and cluster conformations. All values are given in $\mathrm{kcal} / \mathrm{mol}$

\begin{tabular}{ccc}
\hline Geometry & CNT $(5,5)$ & CNT $(6,6)$ \\
\hline unrel. chain & -53.2 & -28.3 \\
unrel. cluster & -21.1 & -34.5 \\
\hline rel. cluster & -25.5 & -34.9 \\
\hline
\end{tabular}

unrelaxed and relaxed structures, where the deformation energy of the $\mathrm{Be}_{4}$ system alone is of $4.4 \mathrm{kcal} / \mathrm{mol}$. In all cases, the difference between counterpoise corrected and uncorrected energies was around $13 \mathrm{kcal} / \mathrm{mol}$, a relatively large number. However, in the case of the linear chain and relaxed cluster, the same calculation was repeated with a larger triple- $\zeta$ basis on the CNT, and the resulting energies were of $-52.0 \mathrm{kcal} / \mathrm{mol}$ and $-26.8 \mathrm{kcal} / \mathrm{mol}$, respectively, with a difference with the uncorrected energies of only $\approx 1.2 \mathrm{kcal} / \mathrm{mol}$. These counterpoised corrected values are in very good agreement with the ones listed in Table 4 , thus validating the calculations using the smaller basis set.

In the case of the the $\operatorname{CNT}(6,6)$, we note that the deformation energy of $\mathrm{Be}_{4}$ (cluster geometry) is of only $0.4 \mathrm{kcal} / \mathrm{mol}$, showing that this structure is much less affected when confined in a larger system, i.e. in a larger cavity with more spatial freedom. The interaction is stronger by approximately $10 \mathrm{kcal} / \mathrm{mol}$ compared to that of the confinement inside the $\operatorname{CNT}(5,5)$, highlighting once again the constraints imposed by the narrower tube. For the chain on the other hand, the interaction energy is $-28.3 \mathrm{kcal} / \mathrm{mol}$, significantly less than in the previous case. However, we should emphasize that $\mathrm{Be}_{4}$ is positioned along the nanotube principal axis, thus this value is certainly lower (in absolute terms) than the optimal one, i.e. the one obtained with the chain closer to the CNT wall, at an optimal adsorption distance. These results allow us to draw some general trends on the practical feasibility to encapsulate a beryllium chain inside a nanotube. In the $\operatorname{CNT}(5,5)$ case, the interaction energy difference of approximately $30 \mathrm{kcal} / \mathrm{mol}$ in favor of the chain over the cluster clearly indicates a preference for the former, such that it seems very unlikely for the chain to fold into the more compact geometry inside the cavity. For the larger nanotube on the other hand, we observe similar interaction energies (despite the uncertainty in the one calculated for the chain) and thus it is hard to discern between the chain and the cluster conformation solely based on thermodynamic data. As a general conclusion to this part, we expect that, at least for narrow nanotubes, the composite system $\mathrm{Be}_{n} @ \mathrm{CNT}(m, l)$ consists of a linear beryllium chain encapsulated inside the carbon nanotube. For nanotubes with a larger diameter, the competition between cluster and linear geometries should instead be studied by a full investigation of the PES, where the particular interactions with the nanotube wall can lead to either structure.

\section{Magnetic Properties of $\mathbf{B e}_{n} @ \mathbf{C N T}(m, l)$}

In this second part of the article we shall discuss the magnetic properties of the composite system $\mathrm{Be}_{n} @ \mathrm{CNT}(m, l)$, but we will first start with a brief summary of what is known about beryllium chains.

The electronic and magnetic properties of isolated linear $\mathrm{Be}_{n}$ systems were already studied in great details by some of this manuscript authors in a series of works employing high level wave function methods $23+26$. The most interesting feature of these systems is the presence of two unpaired electrons localized on both terminal beryllium atoms. These reside in the edge orbitals, like the ones shown in Figure 1 for $\mathrm{Be}_{5}$, and are always antiferromangetically coupled, irrespective of the number $n$ of atoms. In other words, they have a negative value of the magnetic Heisenberg-type coupling constant $J=E_{S}-E_{T}$, where $E_{S}$ and $E_{T}$ correspond to the lowest singlet and triplet energies, respectively. The half-filled edge orbitals give rise to two quasidegenerate states, whose energy split was found to decay exponentially with respect to $n$, i.e. $J \rightarrow 0$ for $n \rightarrow \infty$.

In a more recent study ${ }^{27}$, it was found that beryllium chains deposited on a graphene nanoisland are weakly bonded through dispersion interactions. More interestingly, the magnetic properties of the chain are not lost, but instead strongly influenced by the interaction with the graphene surface: depending on the distance $r$ between the two fragments, the coupling constant $J$ varies and the system switches from antiferromagnetic to ferromagnetic for $r<3.3 \AA$. However, this inversion happens at a distance shorter than the optimal adsorption one, $3.6 \AA$, thus suggesting a difficult practical realization of such a system. Here, following on the idea of the first part of this work, we realize that encapsulating the beryllium chain inside carbon nanotubes can have a similar effect to that observed in Ref. 27. In particular, we expect that the interaction between host and guest systems is such that the choice of nanotube diameter allows to control the strength of the interaction through the distance between the fragments, i.e. the CNT diameter acts as a tunable parameter modulating the coupling of the states. The narrower is the tube, the stronger is the influence on $J$, and viceversa. We thus have two parameters which control the value of $J$ : the length of the chain and the diameter of the CNT. In order to quantify this effect and study the dependence of $J$ on the parameters, a series of CASSCF and NEVPT2 calculations was carried out, whose results are summarized in Table 5 and Table 6 for a number of combinations of chain lengths and nanotube diameters. First note how the interaction with the nanotube

Table $5 J$ coupling constants computed with the $\operatorname{CASSCF}(2,2)$ nanotube for different CNT diameters and chain lengths. Note that the last column refers to the distance between the chain and the nanotube, the CNT diameter is given by $2 d$. All values are given in meV unless otherwise marked

\begin{tabular}{cccc}
\hline host/guest & $\mathrm{Be}_{4}$ & $\mathrm{Be}_{5}$ & $d[\AA]$ \\
\hline $\mathrm{CNT}(5,4)$ & -1.45 & +0.06 & 3.06 \\
$\mathrm{CNT}(5,5)$ & -4.43 & -1.81 & 3.39 \\
$\mathrm{CNT}(6,5)$ & -6.99 & -0.90 & 3.74 \\
$\mathrm{CNT}(6,6)$ & -7.88 & -1.22 & 4.07 \\
isolated & -8.39 & -1.19 & $\infty$ \\
\hline
\end{tabular}

has a similar effect on $J$ as it was observed for the interaction with the graphene nanoisland, on one side supporting our claim and on the other side suggesting a somewhat weak dependence 
Table $6 J$ coupling constants computed with the NEVPT2 nanotube for different CNT diameters and chain lengths. Note that the last column refers to the distance between the chain and the nanotube, the CNT diameter is given by $2 d$. All values are given in meV unless otherwise marked

\begin{tabular}{cccc}
\hline host/guest & $\mathrm{Be}_{4}$ & $\mathrm{Be}_{5}$ & $d[\AA]$ \\
\hline $\mathrm{CNT}(5,4)$ & -4.97 & +0.72 & 3.06 \\
$\mathrm{CNT}(5,5)$ & -14.54 & -6.07 & 3.39 \\
$\mathrm{CNT}(6,5)$ & -19.64 & -2.83 & 3.74 \\
$\mathrm{CNT}(6,6)$ & -20.50 & -3.56 & 4.07 \\
isolated & -16.37 & -2.50 & $\infty$ \\
\hline
\end{tabular}

of $J$ on the curvature of the $\pi$ system. For the narrowest nanotube, with a distance between the chain and the wall of $3.06 \AA$, we observe the most significant change of the magnetic coupling with respect to the isolated chain, where the sign of $J$ flips in the case of $\mathrm{Be}_{5}$ for both methods. Gradually increasing the diameter of the CNT, in other words increasing the distance between the fragments, reduces the interaction and restores an (increasingly stronger) antiferromagnetic coupling. This behavior is most clearly visible for the CASSCF values, Table 5 in particular for $\mathrm{Be}_{4}$ : $J$ monotonically goes from $-1.45 \mathrm{meV}$ to $-7.88 \mathrm{meV}$ as the diameter of the nanotube is increased, approaching from above the value of the isolated chain, i.e. $-8.39 \mathrm{meV}$. By considering the effects of dynamical electron correlation at NEVPT2 level of theory (first column of Table 6), $J$ does not approach anymore the value of the isolated chain in a monotonic manner, but instead the coupling becomes stronger for $\operatorname{CNT}(6,5)$ and $\operatorname{CNT}(6,6)$, and weaker for the other two nanotubes. This same behavior is observed for the longer $\mathrm{Be}_{5}$ chain as well, although the differences are much less pronounced than for $\mathrm{Be}_{4}$. Moreover, here we note that the value corresponding to $\operatorname{Be}_{5} @ \operatorname{CNT}(5,5)$ is clearly off the trend. We can therefore identify two main issues. On one side it is not clear why including dynamical electron correlation makes the coupling $J$ oscillate around the value of the isolated chain, and on the other side, it is not clear the nature of the outlying value for $\mathrm{Be}_{5} @ \mathrm{CNT}(5,5)$.

A possible explanation could lie in the methodology used. It is known that perturbation theory is particularly susceptible to the choice of active space, thus we assessed our choice to use 2 electrons in 2 orbitals by repeating the calculations for the systems $\mathrm{Be}_{4} @ \mathrm{CNT}(5,5), \mathrm{Be}_{4} @ \mathrm{CNT}(6,6)$ and $\mathrm{Be}_{5} @ \mathrm{CNT}(5,5)$ with a larger $(6,6)$ active space, where the extra electrons and orbitals come from the nanotube. As can be seen from Table 7 there is a slight shift of $J$ for $\mathrm{Be}_{4}$ systems, while a much stronger effect is observed for $\mathrm{Be}_{5} @ \mathrm{CNT}(5,5)$, confirming the difficulty encountered with this system and suggesting that the reason for this off-trend value lies somewhere else. On the other hand, the fact that the result did not change for $\mathrm{Be}_{4} @ \mathrm{CNT}(5,5)$ and $\mathrm{Be}_{4} @ \mathrm{CNT}(6,6)$ and that the natural orbital occupation numbers of the extra 4 orbitals are either 1.98 or 0.02 in all systems considered, supports the choice of a $(2,2)$ active space. Since the enlarged active space did not change the overall behavior of $J$, another possible reason for the appearance of the outlier value and an explanation of the NEVPT2 results might come from the fact that we have used nanotubes of
Table $7 \mathrm{~J}$ coupling constants for different CNT diameters and chain lengths obtained with a larger $(6,6)$ active space. All values are given in $\mathrm{meV}$

\begin{tabular}{ccc}
\hline system & CASSCF(6,6) & NEVPT2 \\
\hline $\mathrm{Be}_{4} @ \mathrm{CNT}(5,5)$ & -4.90 & -14.88 \\
$\mathrm{Be}_{4} @ \mathrm{CNT}(6,6)$ & -8.24 & -20.70 \\
$\mathrm{Be}_{5} @ \mathrm{CNT}(5,5)$ & -2.72 & -8.29 \\
\hline
\end{tabular}

finite length. As a matter of fact, finite-size nanotubes with different chiral indices are particularly sensible to the way in which they are terminated (cut from an infinitely long nanotube) and considering the very small range in which $J$ varies, this fact might play an important role. To investigate the effects of the length, we report in Table 8 the magnetic coupling constant for $\mathrm{Be}_{5}$ confined inside the three narrower nanotubes with an extra unit added, making them roughly $2.5 \AA$ longer. The results for the chiral nan-

Table $8 \mathrm{~J}$ coupling constants for $\mathrm{Be}_{5}$ confined in CNTs with an extra unit added. All values are given in $\mathrm{meV}$

\begin{tabular}{cccc}
\hline nanotube & CASSCF(2,2) & NEVPT2 & $d[\AA]$ \\
\hline $\operatorname{CNT}(5,4)$ & -0.06 & +0.06 & 3.06 \\
$\operatorname{CNT}(5,5)$ & +0.11 & +0.48 & 3.39 \\
$\operatorname{CNT}(6,5)$ & -0.93 & -2.96 & 3.74 \\
\hline
\end{tabular}

otubes are only slightly affected by the increased length, whereas $\mathrm{Be}_{5} @ \mathrm{CNT}(5,5)$ still reamins off the trend, although this time in a somewhat less pronounced way. We should note however, that adding only one unit to the nanotube changes the surrounding symmetric pattern given by the carbon structure. To preserve the same bonding topology around the chain, one should in principle add two units, one for each end. This does not seem to play a crucial role for $\mathrm{Be}_{5} @ \mathrm{CNT}(5,4)$ and $\mathrm{Be}_{5} @ \mathrm{CNT}(6,5)$ since the $J$ values are minimally affected, but the opposite seem true for $\mathrm{Be}_{5} @ \mathrm{CNT}(5,5)$. To this end, we calculated $J$ for the latter with yet a longer CNT, restoring the symmetry around the beryllium chain and in this case the values obtained for the coupling were $J=-0.67 \mathrm{meV}$ and $J=-2.55 \mathrm{meV}$ for the CASSCF and NEVPT2 methods, respectively. These values are now fitting well inside the trend observed from Tables 5 and 6. To summarize the cumbersome $\mathrm{Be}_{5} @ \mathrm{CNT}(5,5)$ case, it appears that the original length of the nanotube was causing the odd value of the magnetic coupling. Increasing by one unit the nanotube modified $J$, although the value was still not falling within the general trend, this time probably because the particular pattern of carbon atoms around the chain was different. This pattern is restored by adding a second unit, which finally provided a sensible value of $J$ following the behavior of the other systems. On the other hand, neither enlarging the active space, nor increasing the length of the nanotube have provided an explanation for the oscillating $J$ values obtained at NEVPT2 level of theory. The reason could lie in many of the approximations introduced in this study, for instance the very choice of methodology based on perturbation theory or the fact that the systems are not relaxed.

To summarize this discussion and connect our results to the previous study on $\mathrm{Be}_{n}$ chains deposited on a graphene nanoisland, we 
note that although the sign change of $J$ is observed for the system $\mathrm{Be}_{5} @ \mathrm{CNT}(5,4)$, its magnitude $(J=+0.72)$ is significantly smaller to the one obtained for the interaction of $\mathrm{Be}_{5}$ with the graphene nanoisland, i.e. $J \approx+4.8$. The work by Evangelisti et al. $\frac{27}{\text { high- }}$ lighted how the change of $J$ happens quite rapidly within a limited range of the chain-surface distance. Such a fine control for the confined chain is not possible, assuming that it lies along the CNT central axis. Notice that for the two narrowest nanotubes, the radius is shorter than the adsorption distance observed for the interaction with the graphene fragment, on the other hand, for large nanotubes it is likely that the chain will be adsorbed only on one side of the CNT or form the cluster. At last, we note that a narrower $\operatorname{CNT}(4,4)$ with a diameter of $5.43 \AA$ was also considered as part of this investigation, however the chain-wall distance was too short and the edge orbitals hybridized with the nanotube $\pi$ cloud, essentially destroying the magnetic properties of the chain.

\section{Conclusions}

In this work we have explored the confinement of beryllium chains of different lengths inside carbon nanotubes of different diameters, lengths and helicities. This composite system is interesting for two reasons: on one hand, the carbon nanostructure prevents the chain to fold and form a cluster structure, while on the other hand, the presence of the nanotube significantly affects the magnetic properties of the beryllium chain.

Regarding the former, it was found that although the cluster conformation for both $\mathrm{Be}_{4}$ and $\mathrm{Be}_{5}$ is energetically dozens of $\mathrm{kcal} / \mathrm{mol}$ lower than the linear one, when the $\mathrm{Be}_{n}$ system is confined inside a narrow nanotube, the situation is the opposite. This will remain true as long as the size of the cavity remains small enough as to prevent the chain to fold. In the case of larger nanotubes however, a full investigation of the potential energy surface is required to predict the isomerization pathways between the chain and the cluster structures, since an analysis based on thermodynamics quantities is not sufficient.

Regarding the magnetic properties, there is evidence that the interaction between the chain and the enclosing nanotube modifies the coupling constant $J$, pushing its value towards zero for increasingly narrower nanotubes. For sufficiently small CNTs, we have found that the composite system becomes ferromagnetic, albeit with a very small coupling. A clear trend for the behavior of $J$ was found based on CASSCF calculations as a function of the CNT diameter for both chains considered. The investigation of $J$ with respect to the length of the nanotube was considered for a few selected systems, focusing in particular on $\operatorname{Be}_{5} @ \operatorname{CNT}(5,5)$ : a particularly cumbersome case. It was found for "well-behaved" systems that the results obtained did not change upon addition of an extra unit in the carbon nanotube, while two units where needed for $\mathrm{Be}_{5} @ \mathrm{CNT}(5,5)$. It would clearly be of great interest to investigate longer beryllium chains as well, however this corresponds to use even longer nanotubes, for which the additional computational effort required is at the limit of todays capabilities. To summarize, we have found that the interplay between CNT diameter, length and number of beryllium atoms in the chain can be exploited to create a composite system with tunable magnetic properties. The theoretical and computational challenges to in- vestigate this system are many, and thus in this preliminary work we had to make some tradeoffs. For instance, in the future, relaxation of the complex system should be taken into account in order to allow the chain to go off the principal axis of the nanotube. This should also be complemented by a full study of the potential energy surface to have a more precise prediction about the feasibility of a practical realization of $\mathrm{Be}_{n} @ \mathrm{CNT}(m, l)$.

\section{Conflicts of interest}

There are no conflicts to declare.

\section{Acknowledgements}

The project leading to this publication has received funding from the European Union's Horizon 2020 research and innovation programme under the Marie Skłodowska-Curie grant agreement $\mathrm{n}^{\mathrm{0}} 642294$. S. E. and T. L. acknowledge the "Programme Investissements d'Avenir" ANR-11-IDEX-0002-02, reference ANR10-LABX-0037-NEXT for financial support. N. F.-L. acknowledges Fondazione Cassa di Risparmio di Perugia (P 2014/1255, ACT 2014/6167) for funding. Calculations producing the results presented in this article were carried out using HPC resources from CALMIP (Toulouse), under the grant 2016-p1048.

\section{Notes and references}

1 S. Iijima, Nature, 1991, 354, 56-58.

2 M. S. D. Ado Jorio, Gene Dresselhaus, Carbon Nanotubes, Springer Berlin Heidelberg, Berlin, Heidelberg, 2008, vol. 111, p. 247.

3 P. M. Ajayan, T. W. Ebbesen, T. Ichihashi, S. Iijima, K. Tanigaki and H. Hiura, Nature, 1993, 362, 522-525.

4 B. W. Smith, M. Monthioux and D. E. Luzzi, Nature, 1998, 396, 323-324.

5 J. Lee, H. Kim, S. J. Kahng, G. Kim, Y. W. Son, J. Ihm, H. Kato, Z. W. Wang, T. Okazaki, H. Shinohara and Y. Kuk, Nature, 2002, 415, 1005-1008.

6 R. S. Ruoff, D. C. Lorents, B. Chan, R. Malhotra and S. Subramoney, Science, 1993, 259, 346-348.

7 W. Y. Choi, J. W. Kang and H. J. Hwang, Physical Review B, 2003, 68, 193405.

8 R. C. Che, L.-M. Peng, X. F. Duan, Q. Chen and X. L. Liang, Advanced Materials, 2004, 16, 401-405.

9 Z. Liu, Y. Bando, M. Mitome and J. Zhan, Physical Review Letters, 2004, 93, 095504.

10 L.-J. Li, A. N. Khlobystov, J. G. Wiltshire, G. A. D. Briggs and R. J. Nicholas, Nature Materials, 2005, 4, 481-485.

11 B. Xu and B. C. Pan, The Journal of Physical Chemistry C, 2009, 113, 567-570.

12 I. Garg, H. Sharma, K. Dharamvir and V. K. Jindal, The Journal of Physical Chemistry C, 2010, 114, 18762-18772.

13 M. Smeu, F. Zahid, W. Ji, H. Guo, M. Jaidann and H. AbouRachid, Journal of Physical Chemistry C, 2011, 115, 1098510989.

14 H. Abou-Rachid, A. Hu, V. Timoshevskii, Y. Song and L.-S. Lussier, Physical Review Letters, 2008, 100, 196401. 
15 W. Ji, V. Timoshevskii, H. Guo, H. Abou-Rachid and L. Lussier, Applied Physics Letters, 2009, 95, 021904.

16 S. Battaglia, S. Evangelisti, N. Faginas-Lago and T. Leininger, Journal of Molecular Modeling, 2017, 23, 294.

17 K. Koga, G. T. Gao, H. Tanaka and X. C. Zeng, Nature, 2001, 412, 802-805.

18 Y. Maniwa, H. Kataura, M. Abe, S. Suzuki, Y. Achiba, H. Kira and K. Matsuda, Journal of the Physical Society of Japan, 2002, 71, 2863-2866.

19 K. V. Agrawal, S. Shimizu, L. W. Drahushuk, D. Kilcoyne and M. S. Strano, Nature Nanotechnology, 2017, 12, 267-273.

20 D. Nishide, H. Dohi, T. Wakabayashi, E. Nishibori, S. Aoyagi, M. Ishida, S. Kikuchi, R. Kitaura, T. Sugai, M. Sakata and H. Shinohara, Chemical Physics Letters, 2006, 428, 356-360.

21 M. Hart, E. R. White, J. Chen, C. M. McGilvery, C. J. Pickard, A. Michaelides, A. Sella, M. S. P. Shaffer and C. G. Salzmann, Angewandte Chemie International Edition, 2017, 56, 81448148.

22 M. Šulka, D. Labanc, M. Kováč, M. Pitoňák, I. Černušák and P. Neogrády, Journal of Physics B: Atomic, Molecular and Optical Physics, 2012, 45, 085102.

23 A. Monari, V. Vetere, G. L. Bendazzoli, S. Evangelisti and B. Paulus, Chemical Physics Letters, 2008, 465, 102-105.

24 M. Pastore, A. Monari, C. Angeli, G. L. Bendazzoli, R. Cimiraglia and S. Evangelisti, The Journal of Chemical Physics, 2009, 131, 034309.

25 V. Vetere, A. Monari, A. Scemama, G. L. Bendazzoli and S. Evangelisti, The Journal of Chemical Physics, 2009, 130, 024301.

26 A. Monari, G. L. Bendazzoli and S. Evangelisti, Journal of Chemical Theory and Computation, 2009, 5, 1266-1273.

27 S. Evangelisti, A. Monari, T. Leininger and G. L. Bendazzoli, Chemical Physics Letters, 2010, 496, 306-309.

28 B. O. Roos, P. R. Taylor and P. E. M. Siegbahn, Chemical Physics, 1980, 48, 157-173.

29 C. Angeli, R. Cimiraglia, S. Evangelisti, T. Leininger and J.-P. Malrieu, The Journal of Chemical Physics, 2001, 114, 10252-
10264.

30 C. Angeli, R. Cimiraglia and J.-P. Malrieu, Journal of Chemical Physics, 2002, 117, 9138-9153.

31 C. Angeli, M. Pastore and R. Cimiraglia, Theoretical Chemistry Accounts, 2007, 117, 743-754.

32 T. H. Dunning Jr., The Journal of Chemical Physics, 1989, 90, 1007-1023.

33 Avogadro: an open-source molecular builder and visualization tool. Version 1.2.0, http: / / avogadro.cc/

34 A. Austin, G. A. Petersson, M. J. Frisch, F. J. Dobek, G. Scalmani and K. Throssell, Journal of Chemical Theory and Computation, 2012, 8, 4989-5007.

35 F. Neese, Wiley Interdisciplinary Reviews: Computational Molecular Science, 2017, e1327.

36 F. Neese, F. Wennmohs, A. Hansen and U. Becker, Chemical Physics, 2009, 356, 98-109.

37 F. Weigend, Journal of Computational Chemistry, 2008, 29, 167-175.

38 M. J. Frisch, G. W. Trucks, H. B. Schlegel, G. E. Scuseria, M. A. Robb, J. R. Cheeseman, G. Scalmani, V. Barone, B. Mennucci, G. A. Petersson, H. Nakatsuji, M. Caricato, X. Li, H. P. Hratchian, A. F. Izmaylov, J. Bloino, G. Zheng, J. L. Sonnenberg, M. Hada, M. Ehara, K. Toyota, R. Fukuda, J. Hasegawa, M. Ishida, T. Nakajima, Y. Honda, O. Kitao, H. Nakai, T. Vreven, J. A. Montgomery Jr., J. E. Peralta, F. Ogliaro, M. Bearpark, J. J. Heyd, E. Brothers, K. N. Kudin, V. N. Staroverov, R. Kobayashi, J. Normand, K. Raghavachari, A. Rendell, J. C. Burant, S. S. Iyengar, J. Tomasi, M. Cossi, N. Rega, J. M. Millam, M. Klene, J. E. Knox, J. B. Cross, V. Bakken, C. Adamo, J. Jaramillo, R. Gomperts, R. E. Stratmann, O. Yazyev, A. J. Austin, R. Cammi, C. Pomelli, J. W. Ochterski, R. L. Martin, K. Morokuma, V. G. Zakrzewski, G. A. Voth, P. Salvador, J. J. Dannenberg, S. Dapprich, A. D. Daniels, Ö. Farkas, J. B. Foresman, J. V. Ortiz, J. Cioslowski and D. J. Fox, Gaussian 09 Revision D.01, http://www.gaussian. $\mathrm{COm}$

39 S. F. Boys and F. Bernardi, Molecular Physics, 1970, 19, 553566. 